\title{
Theories of incompleteness
}

\section{Thomas Banchoff}

Holes and Other Superficialities. By Roberto Casati and Achille C. Varzi. MIT Press: 1994. Pp. 253. \$32.50, £26.90.

IN case the potential reader has any doubt about the subject matter of Holes, the book jacket makes it clear - it is riddled with perforations. But what kind of holes are these? How do they differ from the holes in coffee cups, doughnuts and Swiss cheese? What characterizes their tholedness'? How do we recognize a hole? How do we describe a hole?

The authors are fascinated by these challenges. They develop a four-part philosophical theory of holes. How can we define what it means for something to have a hole (ontology)? How can we keep our words straight when we speak of the parts of a hole (mereology)? How can we distinguish and classify different types of holes (topology)? What does it mean to fill a hole (morphology)?

Already on page 7 the authors indicate the criteria against which their work should be evaluated: "A philosophical theory originates from astonishment and is judged on its ability to silence astonishment". Although the book partly succeeds in heightening curiosity about the study of holes, it falls short of astonishing the nonspecialist, and insofar as it complicates descriptions and introduces many terms without adequate explanation, it fails to satisfy completely any curiosity it does stimulate. Many discussions are straightforward and easy to follow; others appear artificial and convoluted. Nonetheless, it raises several subtle questions that encourage the reader to think through his or her own examples, and it does provide the nonspecialist with an insight into the concerns and methodologies of philosophers.

A block of Emmental cheese is one of the author's favourite "hosts" for holes left behind by bubbles that form during the cheesemaking process. A hole totally

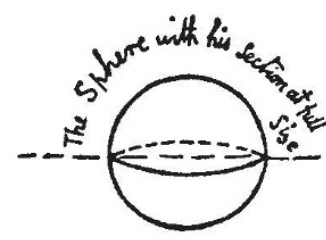
within the block is called a cavity, a hole that meets exactly one of the faces of the block in a single (circular) edge is called a hollow and a large bubble that meets two faces of the block in circular edges is called a tunnel. In a general object, the authors concentrate on hollows and tunnels delimited by edges that are discontinuities in the surface of the host, like the circular edges in the cheese. Moreover, in all the examples in the book, the edges of holes are planar objects.
Unfortunately, these restrictions limit the effectiveness of the theory, and the reader can often be distracted by thinking of examples that don't fit.

Even for a block of Swiss cheese, it is not easy to see how the theory can deal with a bubble that cuts out a piece of an edge, or one that cuts off a corner. One of the key notions is that a hole is something that can be filled. We know how to fill a cavity, a tunnel or a hole in the cheese, but how do we fill these other holes? How do we fill the hole in a cup with a wavy lip or in a somewhat warped doughnut? How do we know where the hole begins? This is a crucial problem if, as the authors suggest, the essence of a hole is dependent on the way it meets the surface of its host (the superficiality referred to in the subtitle).

As a mathematician with a special interest in the geometry of lower and higher dimensions, I was initially pleased to see a reference to my favourite book, Flatland by Edwin A. Abbott (published in 1884, not 1882 as stated; and from which the pictures here are taken). It is a standard practice of geometers to develop concepts in ordinary space by first treating the analogous concepts in the plane. Unfortunately, the authors adopt a materialist attitude that prevents them from empathizing fully with the position of $\mathrm{A}$ Square, the planar protagonist of Flatland, investigating the analogue of a piece of

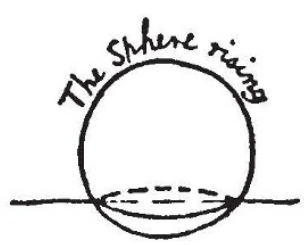
Swiss cheese in his own dimension (represented, in some theories, by the top surface of the three-dimensional block of cheese, or some infinitesimally thin slice or cross-section of the block). A Square would argue that a circular bubble within the (square) slice is a cavity because it has two boundary (curve) pieces, analogous to the distinguishing feature of a cavity in three-space. The authors, however, persist in looking at this hole from a three-space perspective and consider it to be a tunnel because they can

sophical problems of 'emptiness'. In defining holes purely in terms of what is missing, the authors consider holes to be "immaterial bodies". To a mathematician, the situation is simpler. In any dimension, a convex object is defined by the property that any segment determined by two points of the object consists entirely of points of the object. Any object is contained in a smallest convex object, called its convex hull. Holes, then, are certain (maximally connected) pieces of the convex hull that are not parts of the original object. So a hole is a set of points just as the host is. There is nothing mysterious or 'immaterial' about the hole from this geometric viewpoint, although the reader will find many discussions of related philosophical problems in the book.

The diagrams in the book generally represent three-dimensional entities, so they are usually presented with some shading and perspective. Occasionally the authors use two-dimensional slices to illustrate their points, and this can lead to confusion in interpretation, as in the illustrations of a "perfect filling" of a hole and of a "canonical (non-privileged)" fillable part of a hole. There is no obvious way to visualize these as three-dimensional objects, and the concepts "canonical" and "privileged" are never properly defined.

Frequently the philosophical analysis causes the authors to deviate from common usage. For example, they state: "Holes can move, and regions of space cannot". Why? An example will illustrate the process.

In a glass of water with a thick layer of oil on the top, as a bubble rises from the water to the interface between water and oil and finally into the oil, then in ordinary language one might say that it is the same bubble. On the other hand, in the language developed by the authors the bubble creates four different holes during its migration: first a cavity in the water; then two holes, a hollow in the water and another hollow in the oil; and finally a cavity in the oil.

The book ends with 66 or so theorems listed in an appendix along with various definitions and axioms. Despite this formalistic conclusion, the authors try to write for a general audience, and although they lapse into the jargon of one discipline or another at the ends of various chapters, it is always possible to pick up a fresh thread in the next. It is not, however, too easy to skip around: ideas are developed progressively and an innocent-sounding word may earlier have been given a precise, unexpected definition. being who would consider a three-space cavity to be a tunnel from a four-space perspective

The use of mathematical ideas of convexity and geometrical complements can go a long way towards avoiding philo-
Thomas Banchoff is in the Department of Mathematics, Brown University, Providence, Rhode Island 02912, USA. 